\title{
Thermovoltages in vacuum tunneling investigated by scanning tunneling microscopy
}

\author{
D.H. Hoffmann ${ }^{a}$, A. Rettenberger ${ }^{a}$, J.-Y. Grand ${ }^{a}$, K. Läuger ${ }^{a}$, P. Leiderer ${ }^{a}$, K. Dransfeld ${ }^{a}$, R. Möller ${ }^{h}$ \\ "Fakultät für Physik, Universität Konstanz, Postfach 5560, D-78434 Konstanz, Germany \\ ${ }^{\mathrm{b}}$ Physikalisches Institut, Universität Stuttgart, Pfaffenwaldring 57, D-70550 Stuttgart, Germany
}

\begin{abstract}
By heating the tunneling tip of a scanning tunneling microscope the thermoelectric properties of a variable vacuum barrier have been investigated. The lateral variation of the observed thermovoltage will be discussed for polycrystalline gold, stepped surfaces of silver, as well as for copper islands on silver.
\end{abstract}

Keywords: Local density of states; Monoatomic steps; Scanning tunneling microscopy; Thermovoltage

The first experimental evidence for thermovoltages across a tunneling barrier was given by Williams and Wickramasinghe [1] who investigated by scanning tunneling microscopy in air the voltage induced by heating the $\mathrm{MoS}_{2}$ sample. They found a lateral variation between the position of the sulfur and the molybdenum atoms. To explain these findings Støvneng and Lipavsky [2] proposed a theory which relates this thermovoltage to the logarithmic derivative of the electronic density of states close to the fermi energy of the tip and the sample. The measurements which will be presented in this publication are based on an experimental technique making use of the thermal noise in electronic tunneling. As discussed previously [3] the latter is proportional to the square root of the tunneling conductance hence it depends exponentially on the distance between tip and sample. By monitoring the noise amplitude this distance can be controlled in the absence of a direct tunneling current. The latter is canceled by a second feedback loop providing the appropriate external voltage which equals the induced thermal voltage. Details of this method have been described elsewhere [4].

The experiments have been performed in ultrahigh vacuum, and temperature differences of $1-10 \mathrm{~K}$ have been obtained by heating the tunneling tip by laser irradiation of $5-50 \mathrm{~mW}$. Since the laser spot is focused onto the shaft of the tip at a distance of about $1 \mathrm{~mm}$ from the tunneling gap illumination of the gap is excluded. A sketch of the experimental arrangement is shown in Fig. 1. The samples have been prepared in situ by evaporating noble metals onto glass or mica substrates. The tunneling tips have been made of tungsten by electrochemical etching and have been cleaned in situ by field desorption shortly before the measurements.

Fig. 2 shows the topography of a polycrystalline gold film and the simultaneously measured thermovoltage. The latter ranges between $-90 \mu \mathrm{V}$ and $+10 \mu \mathrm{V}$ for an estimated temperature difference of $1 \mathrm{~K}$. The complicated structure is not readily understood, however a detailed investigation suggests that it is correlated to the crystallographic orientation of the gold surface.

To elucidate this assumption epitaxially grown (111) surfaces of silver and copper on mica have been investigated. For a given tunneling tip, in fact, only

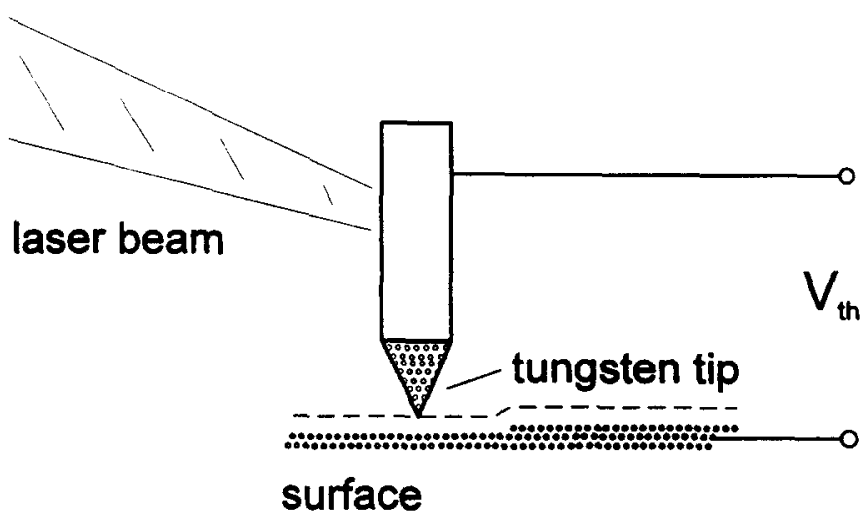

Fig. 1. Scheme of the experimental set-up. 

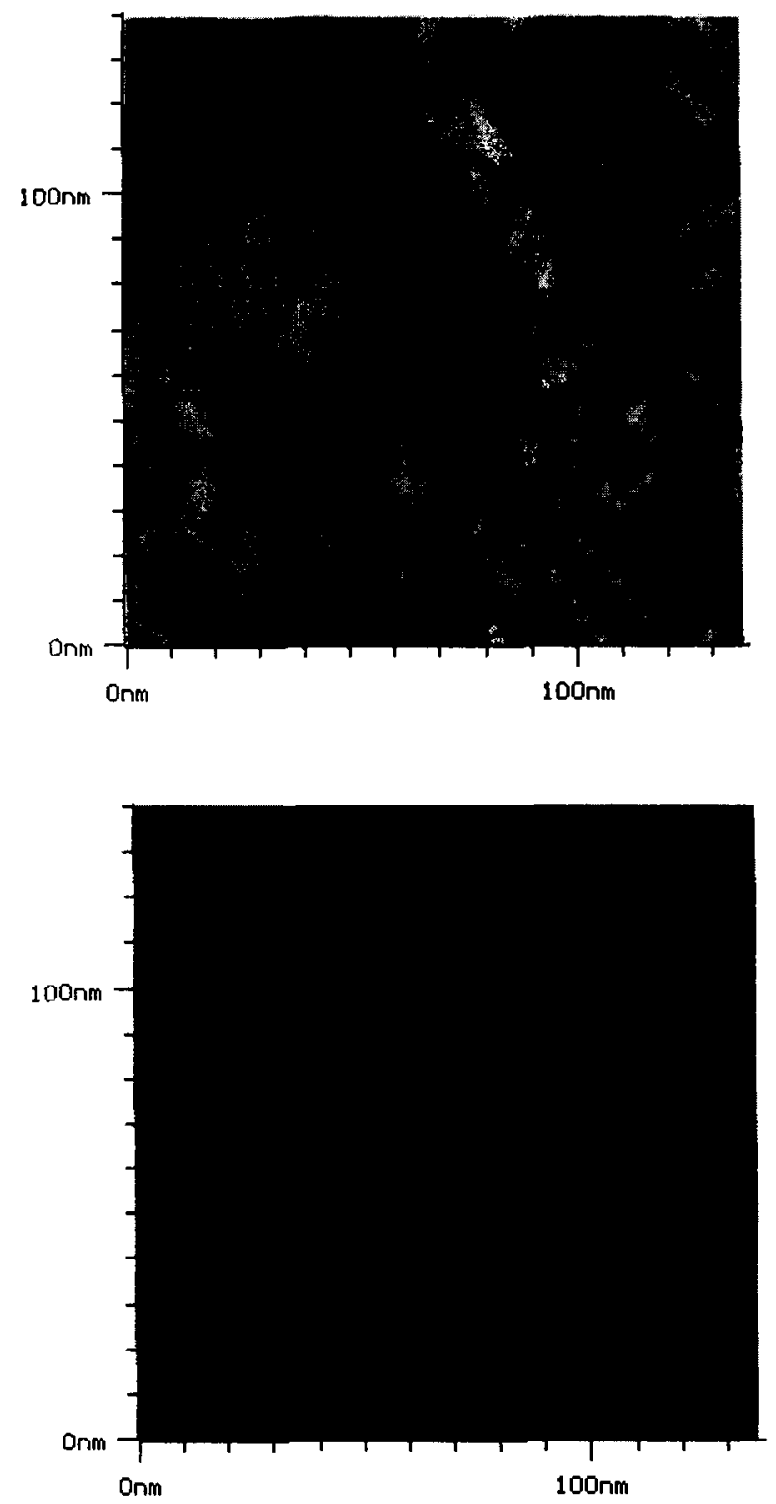

Fig. 2. (a) Topographic image of a polycrystalline gold film, the height difference is about $200 \AA$, the average current noise amplitude amounts to $3 \mathrm{pA}$. (b) Thermovoltage map, the voltage ranges between $-90 \mu \mathrm{V}$ (black) and $+10 \mu \mathrm{V}$ (white) for a temperature difference between tip and sample of about $1 \mathrm{~K}$.

one value of the thermovoltage has been found for a surface without defects. However, the slightest defect yields a pronounced variation. Results for $\mathrm{Ag}(111)$ are shown in Fig. 3. The observed thermovoltage vanishes if the tip is not heated and it is independent of the scan direction and scan speed. Every monoatomic step is connected to an increase of about $20 \mu \mathrm{V}$. This feature extends about $20 \AA$ perpendicular to both sides of the step. Since the steps are much better localized this width has to be corrclated to the physical origin of the thermovoltage.

To study the influence of the sample material copper islands on $\mathrm{Ag}(111)$ have been investigated. The islands can clearly be recognized in the topo-
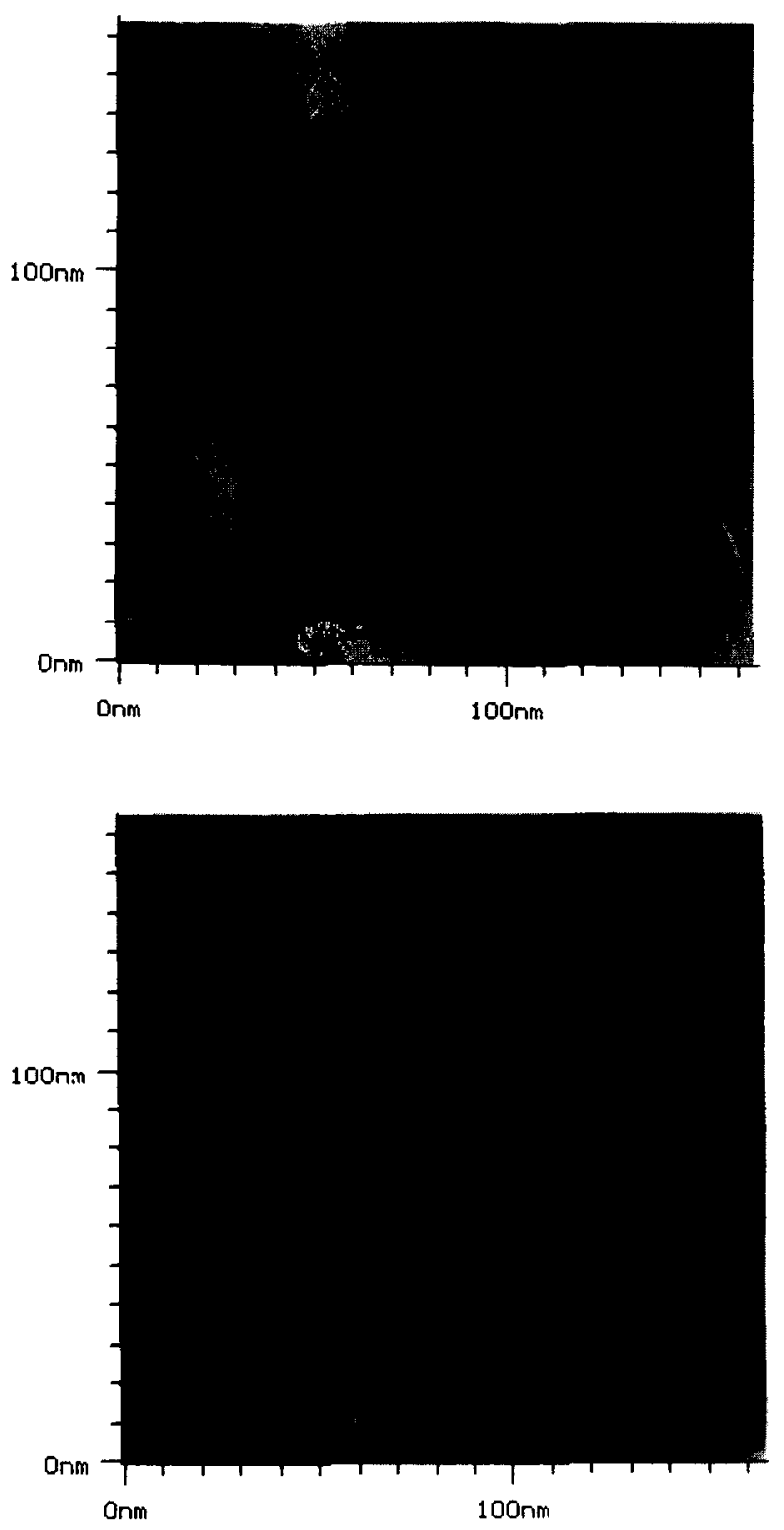

Fig. 3. (a) Topographic image of $\mathrm{Ag}(111)$ on mica, the sample of $4000 \AA$ thickness has been prepared at a substrate temperature of $280^{\circ} \mathrm{C}$ (evaporation rate, $5 \AA \mathrm{s}^{-1}$ ). The height difference is about $150 \AA$, the average current noise amplitude amounts to $5 \mathrm{pA}$. (b) Thermovoltage map, the voltage ranges between $-400 \mu \mathrm{V}$ (black) and $-100 \mu \mathrm{V}$ (white) for a temperature difference between tip and sample of about $5 \mathrm{~K}$. The potential increases at a monoatomic step by about $20 \mu \mathrm{V}$ and is constant on a flat $\mathrm{Ag}(111)$ plane ( $V=$ $-200 \mu \mathrm{V})$.

graphy by their round shape as can be seen in Fig. 4(a). The corresponding thermovoltage is shown in Fig. 4(b). Although the islands have no well-defined orientation of the surface the thermovoltage varies only little on the islands but is significantly different from the $\mathrm{Ag}(111)$ substrate.

Our observation can be understood qualitatively on the basis of the theory of Støvneng, if in addition the contribution of the different $k$ vectors of the electrons is weighted according to their contribution to the 

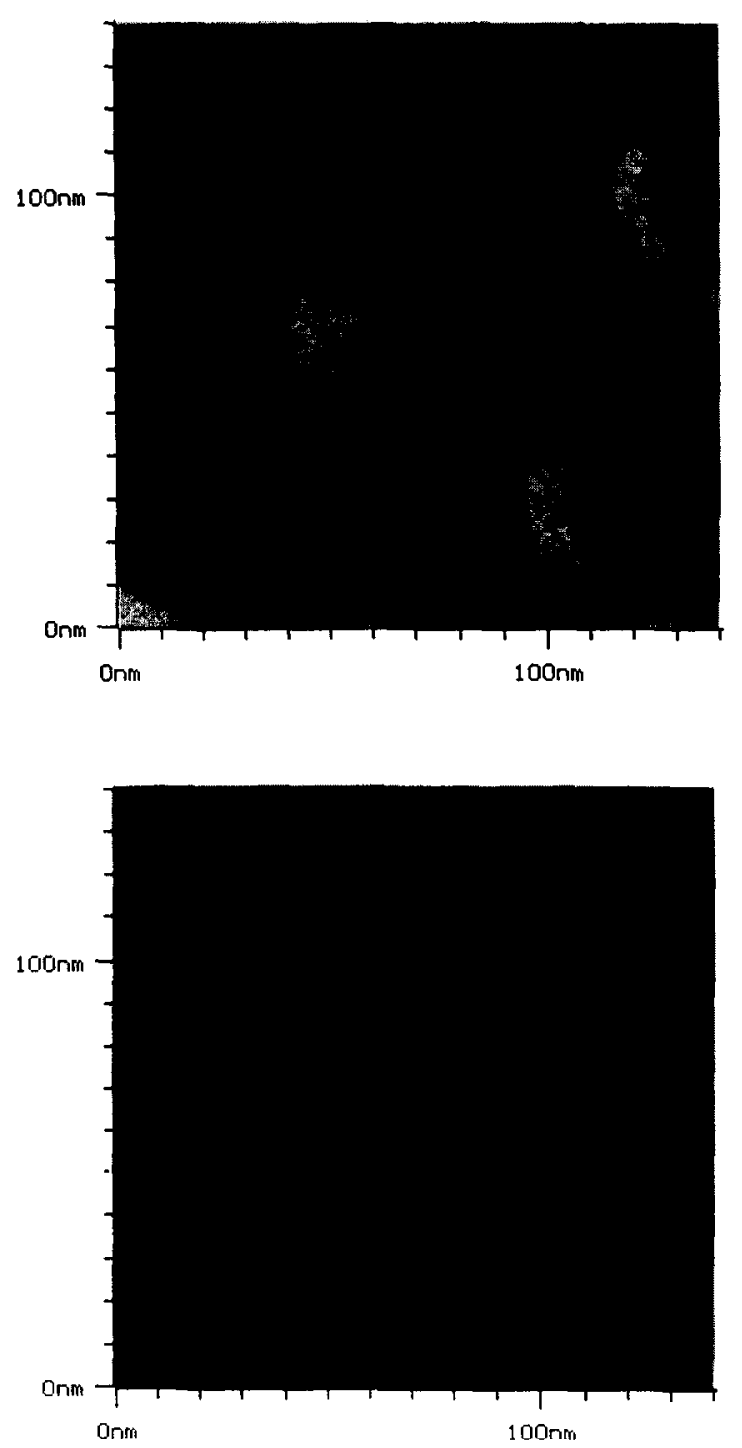

Fig. 4. (a) Topographic image of copper islands on a $\mathrm{Ag}$ (111) substrate covered by $2 \mathrm{MI}$, of $\mathrm{Cu}$. The height difference is about $100 \AA ̊$, the average current noise amplitude amounts to $5 \mathrm{pA}$. (b) Thermıvoltage map, the voltage ranges between $-400 \mu \mathrm{V}$ (black) and $-100 \mu \mathrm{V}$ (white) for a temperature difference between tip and sample of about $7 \mathrm{~K}$.

tunneling process. This may lead to the observed dependence of the thermovoltage on the orientation of the surface. The particular role of the $\mathrm{Ag}(111)$ surface becomes clear since this surface has a band gap for $k_{\text {parallel }}=0$. Recent experiments [5] reveal that a minor contribution to the thermovoltage can be caused by surface states which become visible by the interference of these states at step edges etc. [6].

In conclusion, the voltage across a vacuum tunneling barrier induced by a temperature difference between both electrodes has been investigated. The measurement has been performed by a scanning tunneling microscope with a heated tip. The voltage can be evaluated by operating the microscope in a potentiometry mode at zero bias. To learn about the origin of the thermal-induced voltage its lateral variation has been analyzed for various metallic surfaces. The results on polycrystalline surfaces of gold imply that the signal is correlated to the crystallographic orientation of the area of the sample facing the tunneling tip. On $\mathrm{Ag}(111)$ and $\mathrm{Cu}(111)$ the signal shows a variation at monoatomic steps which extends about $20 \AA$ to each side of the step. A heterogeneous surface with copper islands on $\mathrm{Ag}(111)$ reveals a clear contrast between the substrate and the islands.

\section{Acknowledgments}

The authors would like to thank the Deutsche Forschungsgemeinschaft (SFB306) for financial support and $\mathrm{C}$. Baur for invaluable help during the experiments.

\section{References}

[1] C.C. Williams and H.K. Wickramasinghe, Nature, 344 (1990) 317-319.

[2] J.A. Støvneng and P. Lipavsky, Phys. Rev. B, 42 (14) (1990) 9214-9216.

[3] R. Möller, A. Esslinger and B. Koslowski, Appl. Phys. Lett., 55 (22) (1989) 2360-2362.

[4] R. Möller, C. Baur, A. Esslinger, U. Graf and P. Kürz, J. Vac. Sci. Technol. B, 9 (2) (1991) 609-611.

[5] D. Hoffmann, A. Rettenberger, K. Läuger, J.Y. Grand and R. Möller, to be published.

[6] M.F. Crommie, C.P. Lutz and D.M. Eigler, Nature, 524 (1993) 363. 